\title{
Evidence for Rayleigh-Taylor Plasma Instability at the Front of Solar Coronal Mass Ejections
}

\author{
Daniele Telloni $^{1, * \mathbb{D}}$, Francesco Carbone ${ }^{2} \mathbb{D}$, Alessandro Bemporad ${ }^{1}(\mathbb{D})$ and Ester Antonucci ${ }^{1}$ (D) \\ 1 National Institute for Astrophysics, Astrophysical Observatory of Torino, Via Osservatorio 20, \\ 10025 Pino Torinese, Italy \\ 2 National Research Council, Institute of Atmospheric Pollution Research, c/o University of Calabria, \\ 87036 Rende, Italy \\ * Correspondence: daniele.telloni@inaf.it; Tel.: +39-011-8101-984
}

Received: 26 July 2019; Accepted: 12 August 2019; Published: 15 August 2019

\begin{abstract}
This work focuses on the interaction of a Coronal Mass Ejection (CME) with the ambient solar corona, by studying the spatial and temporal evolution of the density fluctuations observed by the SOHO/UV Coronagraph Spectrometer (UVCS) during the CME. The investigation is performed by applying a wavelet analysis to the HI Ly $\alpha 1216 \AA$ line intensity fluctuations observed with UVCS during the CME occurred on 24 December 2006. Strong and coherent fluctuations, with a significant spatial periodicity of about $84 \mathrm{Mm} \simeq 0.12 \mathrm{R}_{\odot}$, are shown to develop in about an hour along the front of the CME. The results seem to indicate the Rayleigh-Taylor (RT) instability, susceptible to the deceleration of the heavier fluid of the CME front into the lighter surrounding coronal plasma, as the likely mechanism underlying the generation of the observed plasma fluctuations. This could be the first inference of the RT instability in the outer solar corona in UV, due to the transit of a CME front in the quiet coronal plasma; this interpretation is also supported by a linear magnetohydrodynamic analysis of the RT instability.
\end{abstract}

Keywords: sun: corona; sun: coronal mass ejections (CMEs); sun: oscillations; instabilities; plasmas; turbulence

\section{Introduction}

During Coronal Mass Ejections (CMEs) coronal plasma is expelled against the gravitational field of the Sun into the interplanetary space, over the course of minutes to hours. Each CME drains solar mass in the range between $10^{11}$ and $10^{13} \mathrm{~kg}$ [1] and expands at speeds ranging from 100 to $2500 \mathrm{~km} \mathrm{~s}^{-1}$ e.g., [2], thus with a kinetic energy of the order of $10^{21}-10^{25} \mathrm{~J}$. CMEs carry also magnetic, thermal and nonthermal energies. In particular, it is worth noting that during the primary dissipation processes (associated to magnetic reconnection) most of the free magnetic energy (about 68\%) is used to accelerate electrons and ions (nonthermal energy), while only a relatively small portion of the magnetic energy (about 7\%) goes into CME kinetic and gravitational potential energies (see the review by [3], and references therein).

As CMEs propagate outward, they interact with the ambient coronal plasma, producing a variety of effects, such as the formation of fast-mode forward shocks ahead of the CME e.g., $[4,5]$, which heat the solar wind e.g., [6] and accelerate Solar Energetic Particles (SEPs) [7]. Type II solar radio bursts, likely associated to the compression between the CME and the unperturbed coronal plasma, have been recently observed to originate also at the flanks of CMEs e.g., [8,9]. During CMEs expansion, magnetic reconnection events can occur e.g., [10,11], in particular, along their flanks with the surrounding coronal magnetic field, causing secondary small-scale plasma ejections [12]. The interaction of a CME with the coronal plasma can in general lead to a restructuring of the overall 
magnetic configuration of the corona [13]. Recently, vortexes were observed along the flanks of CMEs in the images obtained with the Atmospheric Imaging Assembly (AIA) on board the Solar Dynamics Observatory (SDO) [14,15], interpreted in terms of Kelvin-Helmholtz instability.

This paper presents evidence for non-stationary density fluctuations induced at the interface of a CME with the surrounding plasma during its outward propagation, and provides further information on the dynamic and energetic interaction of CMEs with the ambient corona. The characteristics attributed to the plasma density fluctuations allow the identification of the physical mechanisms underlying the release of energy and momentum from the ejecta to the ambient coronal wind.

This study is performed by applying a wavelet analysis to the time series of the intensity of the HI Ly $\alpha 1216 \AA$ line observed by UVCS, the UltraViolet Coronagraph Spectrometer [16] on board the Solar and Heliospheric Observatory (SOHO), during the CME event occurred on 24 December 2006 at the South pole in a coronal hole region. The information derived from the wavelet transform on both amplitude and phase of the temporal fluctuations allows the visualization of the region of the CME interacting with the ambient corona and a measurement of the energy transferred to the coronal plasma.

The layout of the paper is as follows: description of the observations (Section 2), description of the methodological approach to the data and presentation of the data analysis (Section 3), discussion (Section 4) and interpretation of the results (Section 5), conclusions (Section 6).

\section{Observations}

The CME event of 24 December 2006 was observed with the UVCS instrument on board the SOHO spacecraft above the south-west limb. The HI Ly $\alpha$ line, emitted by the neutral hydrogen atoms at $1215.67 \AA$, was continuously detected, throughout the event, in the field of view (FOV) of the spectrometer. Its entrance slit was set at $1.87 \mathrm{R}_{\odot}$ from the centre of the Sun and oriented perpendicularly to the radial direction, in such a way to cover a broad latitude range, from $170.5^{\circ}$ to $212.5^{\circ}$ (counterclockwise from the North pole). The slit was delimiting a FOV $\left(22^{\prime} \times 84^{\prime \prime}\right)$ wide, divided in 46 spatial bins with a spatial scale of $28^{\prime \prime} \sim 0.03 \mathrm{R}_{\odot}$ per bin. The entire set of observations consisted of $N=2324$ exposures acquired with a cadence time of $\delta t=2 \mathrm{~min}$, in the interval from 07:27 UT to 15:40 UT, encompassing the entire CME event.

The CME front first appeared above $2 \mathrm{R}_{\odot}$ in the FOV of LASCO, the SOHO Large Angle Spectrometric Coronagraph [17] at 15:54 UT, expanding in the southward direction. Figure 1a-e show the LASCO C2 white light images during the passage of the CME. These images are obtained by subtracting the last one acquired before the CME detection. The classical three-part structure of coronal mass ejections (i.e., a bright arch-shaped front including a darker cavity and a bright core) cannot be clearly identified in the LASCO images. The propagation of the CME front is described in Figure $1 \mathrm{f}$, where the height of the front is plotted as a function of time, thus allowing the measurement of the expansion speed and acceleration of the CME.

The synoptic map obtained by subtracting the pre-CME intensity from the HI Ly $\alpha$ line emission, observed with UVCS, shows the transit of the mass ejection front through the spectrometer slit Figure 2. The zonal average of the HI Ly $\alpha$ intensity (Figure 2b) outlines the evolution of the CME: a first sharp peak around 15:14 UT is followed by a further more gradual increase up to a second intensity peak around 17:26 UT, and a slow decrease lasting several hours. The duration of the CME passage through the UVCS slit is about $10 \mathrm{~h}$, including the growth time of the UV light curve to its most intense peak and the 1/e-relaxation time. The front of the CME crosses the UVCS slit in about one hour. The first and second peaks are associated with the transit of the front and the core of the ejected plasma, respectively. Thus, the general three-component structure of CMEs, not observable in visible light above $2 R_{\odot}$, is partially observed with UVCS at lower heliodistance, hence closer to the source. 

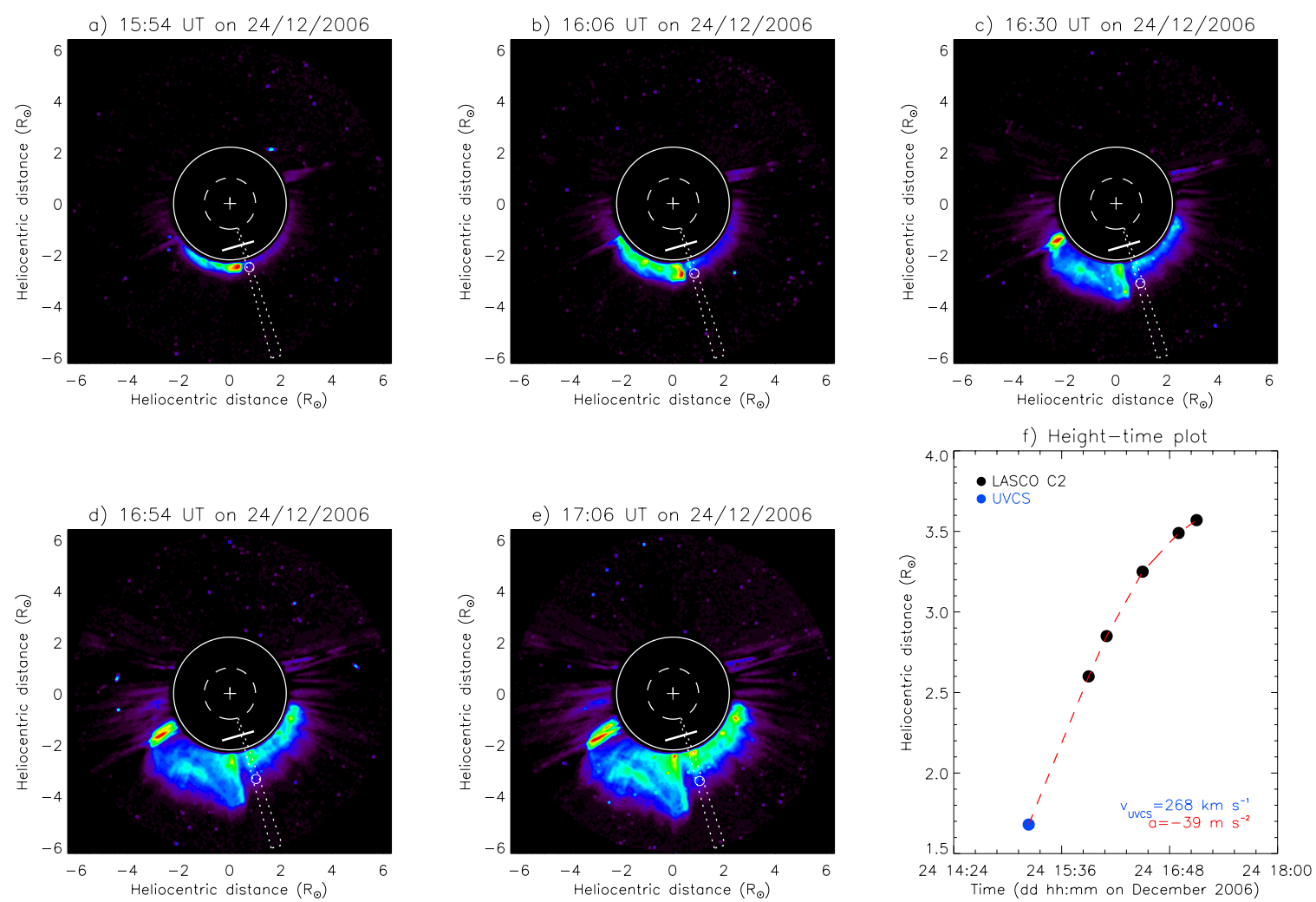

Figure 1. (a-e) LASCO C2 base difference images showing the white light corona as observed during the transit of the CME front through the UVCS slit (shown as a white thick line) at 15:54, 16:06, 16:30, 16:54 and 17:06 UT on 24 December 2006; the dashed white line indicates the solar limb, whilst the continuous one shows the LASCO C2 occulter; the dotted area marks the coronal region where the CME front is identified (open circle). (f) Height-time plot of the CME front: black and blue dots correspond to the LASCO C2 and UVCS observations, respectively; the parabolic fit (red curve) indicates a deceleration of the CME of $\sim-39 \mathrm{~m} \mathrm{~s}^{-2}$ after 15:14 UT and an expansion speed of $\sim 268 \mathrm{~km} \mathrm{~s}^{-1}$ at the UVCS position.

After 15:14 UT the CME is slowing down with a constant deceleration of about $-39 \mathrm{~m} \mathrm{~s}^{-2}$, as inferred by means of a parabolic fit of the height-time plot of Figure $1 \mathrm{f}$, from an initial expansion speed of $\sim 268 \mathrm{~km} \mathrm{~s}^{-1}$ measured at the UVCS slit position. The propagation direction of the region of the CME observed with UVCS is inferred to be primarily in the plane of the sky, since no significant Doppler shift of the emission of the HI Ly $\alpha 1216 \AA$ line was detected. This implies that the values of $\sim 268 \mathrm{~km} \mathrm{~s}^{-1}$ and $-39 \mathrm{~m} \mathrm{~s}^{-2}$ correspond to the expansion speed and deceleration of the coronal plasma observed with UVCS along its direction of propagation. 


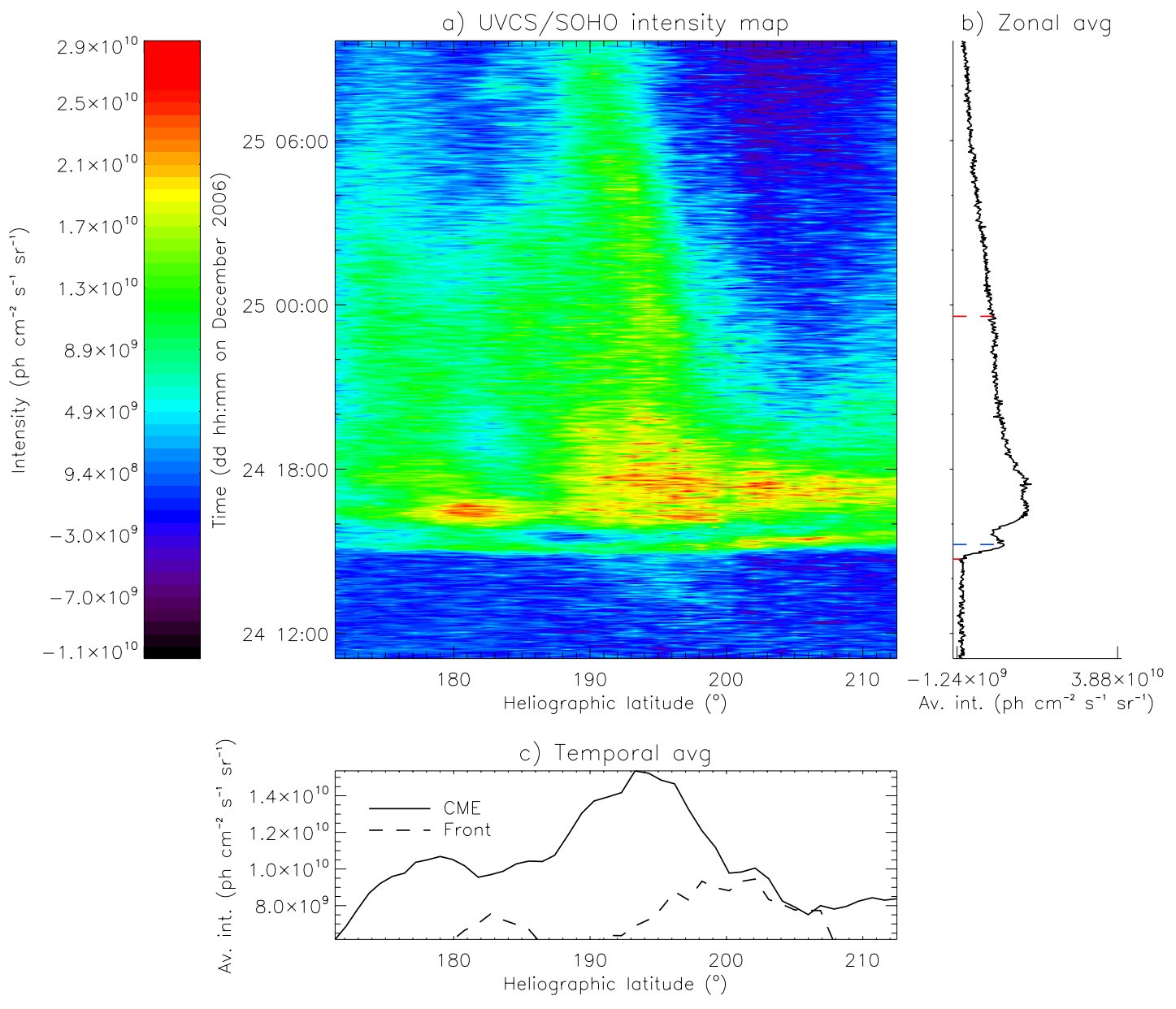

Figure 2. (a) Synoptic map of the HI Ly $\alpha$ line intensity as a function of time and heliographic latitude (expressed in degrees counterclockwise from the North pole). (b) Zonal average of (a), where the duration of the CME and the arrival time of the CME front (15:14 UT) are indicated by red and blue dashed lines, respectively. (c) Temporal average of (a) within the whole CME event (solid line) and at the CME front, that is from 14:42 UT to 15:30 UT on 24 December 2006 (dashed line).

\section{Analysis and Results}

The analysis of the HI Ly $\alpha$ emission detected during the CME is performed by means of a complex wavelet transform in order to identify periodic components of non-stationary signals in the input data and to determine both the dominant modes of variability and how those modes evolve in time, by decomposing a time series into time-frequency space. By combining the results achieved at multiple locations, the spatial and temporal variability of a field of data can be reconstructed. Furthermore, the phase information inferred by applying the wavelet transform, quantifies the degree of coherence between adjacent oscillation patterns.

The wavelet technique, increasingly adopted in astrophysical studies, was used for instance to detect (i) outward propagating perturbations with periods of $180-420 \mathrm{~s}$ at a footpoint of an active region bright loop [18], (ii) a sporadic oscillation with periods of 7-8 $\mathrm{min}$ in the quiet solar corona [19], (iii) coherent fluctuations within a weak solar magnetic network region [20] and (iv) magnetic and cross-helicity events in the solar wind [21,22]. In the present paper, this method is applied to explore the region of interaction between a CME and the surrounding corona in order to investigate the existence of oscillatory signals associated with the transit of the ejected plasma. 


\subsection{Wavelet Amplitude Spectrum}

The wavelet transform is applied to the time series of the HI Ly $\alpha 1216 \AA$ line intensity, detected in each of the 46 bins of the UVCS slit, $I_{L y \alpha}\left(\phi_{i}, t\right)$, with $\phi_{i}$ ranging from $\phi_{1}=170.5^{\circ}$ to $\phi_{46}=212.5^{\circ}$, counterclockwise from the North pole and $t=0, \ldots,(N-1) \delta t$. The long uninterrupted time series and the short sampling time $\delta t=2 \mathrm{~min}$ allow the investigation of a broad range of periods ranging from the smallest resolvable one $T_{N S}=2 \cdot \delta t=4 \mathrm{~min}$, the Nyquist-Shannon period, up to $(N-1) \delta t \simeq 77 \mathrm{~h}$.

The removal from each $I_{L y \alpha}\left(\phi_{i}, t\right)$ data-set of the low-frequency power associated with the CME structure, is performed by applying a high-pass band filter, in order to allow the detection of high-frequency fluctuations in the interaction region. The high-pass filter removes, in addition, the frequency contribution due to solar rotation and the large-scale background fluctuations, exhibiting a $f^{-2}$ power law, observed at the poles with UVCS [23,24]. A similar approach has been used, for instance, by Dolla et al. [25] when studying quasi-periodic pulsations during the impulsive phase of solar flares. For each $I_{L y \alpha}\left(\phi_{i}, t\right)$ intensity series the width of the filter window varies along the time axis: a narrower window is applied when the signal evolves more rapidly (i.e., during the passage of the CME front), whilst a broader window is chosen when the intensity is varying more slowly (i.e., during the relaxation time of the coronal plasma after the CME transit). In this way, the overall trend of the CME signal is accurately removed in each pixel. Moreover, since the window width is not the same for a given time series, the introduction of spurious frequencies associated with the choice of the width is avoided. In Figure 3, the HI Ly $\alpha$ intensity detected in pixel 34 of the UVCS slit, at 201 ${ }^{\circ}$, is shown, as an example, before (black curve) and after (red curve) the filtering process; as expected, the overall CME signal has been removed in the resulting filtered data-set $\tilde{I}_{L y \alpha}\left(\phi_{34}=201^{\circ}, t\right)$.

Given a wavelet function, $\Psi_{0}(\eta)$, depending on a non-dimensional 'time' parameter $\eta$, with zero mean and localized both in time and frequency space, the continuous wavelet transform of the discrete time series $\tilde{I}_{L y \alpha}\left(\phi_{i}, t\right)$ is defined as the convolution of $\tilde{I}_{L y \alpha}\left(\phi_{i}, t\right)$ with a scaled and translated version of $\Psi_{0}(\eta)[26]$ :

$$
W\left(\phi_{i}, t, s\right)=\frac{1}{\sqrt{N}} \sum_{t^{\prime}=0}^{(N-1) \delta t} \tilde{I}_{L y \alpha}\left(\phi_{i}, t^{\prime}\right)\left(\frac{\delta t}{s}\right) \Psi_{0}^{*}\left(\frac{t^{\prime}-t}{s}\right),
$$

where the asterisk indicates the complex conjugate and $\Psi_{0}(\eta)$ is normalized to have unit energy. By varying the wavelet scale $s$ and by translating the wavelet function $\Psi_{0}(\eta)$ in the time domain, it is possible to quantify the amplitude of any oscillatory features as a function of both the spatial scale and time. It is worth noting that the wavelet scale $s$ does not necessarily correspond to the Fourier period, $\tau$, because of the functional form of the wavelet function. The relationship between the equivalent Fourier period and the wavelet scale is derived analytically for a particular wavelet function by substituting a cosine wave of a known frequency $v=\tau^{-1}$ into Equation (1) and computing the scale $s$ at which the wavelet power spectrum peaks.

The wavelet function $\Psi_{0}(\eta)$ that is best suited to the data depends on the aim of the study. The choice of the wavelet function depends on several factors: its shape and width, and whether it is complex or real valued. Complex wavelets, as the Paul and Morlet functions, are the ideal candidates for this study, since they yield complex wavelet transforms that can be divided into amplitude $\left|W\left(\phi_{i}, t, s\right)\right|$ and phase $\tan ^{-1}\left[\mathfrak{I} W\left(\phi_{i}, t, s\right) / \mathfrak{R} W\left(\phi_{i}, t, s\right)\right]$, thus allowing the evaluation of power and degree of coherence of any oscillatory features. Moreover, being damping cosine wavelets, the Paul and Morlet functions are suitable to be applied to smoothly varying time series, such as the HI Ly $\alpha$ intensity $\tilde{I}_{L y \alpha}\left(\phi_{i}, t\right)$. The width of the wavelet function, namely the $e$-folding time of the its amplitude, is correlated to the resolution of the wavelet itself: a narrow function in time, as the Paul wavelet, has good time resolution but poor frequency resolution, whilst a broader function, as the Morlet wavelet, has good frequency resolution at the expenses of the time resolution. Since the main aim of this study is to ascertain the existence and identify the time of occurrence of quasi-periodic oscillations associated with the propagation of the CME in the ambient coronal plasma, rather than high resolution of the various periods involved in the process, the Paul wavelet function, which has a better capability 
(with respect to the Morlet) of detecting the time occurrence of a given periodicity, has been used throughout the analysis shown in this paper. The Paul wavelet function is given by:

$$
\Psi_{0}(\eta)=\frac{2^{m} i^{m} m !}{\sqrt{\pi(2 m) !}}(1-i \eta)^{-(m+1)},
$$

where $m=4$ is the order of the wavelet. The width is $s / \sqrt{2}$ and the wavelet scale $s$ is related to the Fourier period $\tau$ by the expression $\tau=\frac{4 \pi}{2 m+1} s=1.4 s$.

Figure $3 \mathrm{~b}$ shows as an example the wavelet amplitude spectrum, $\left|W\left(\phi_{34}=201^{\circ}, t, s\right)\right|$, for the filtered data-set $\tilde{I}_{L y \alpha}\left(\phi_{34}=201^{\circ}, t\right)$ (red curve of Figure 3a). The ordinate represents the Fourier period $\tau$, to which the scales $s$ have been converted, the abscissa corresponds to the time of observation. The thick contour lines correspond to the $95 \%$ confidence level determined on the basis of a null hypothesis. That is, in the hypothesis that $\tilde{I}_{L y \alpha}\left(\phi_{i}, t\right)$ is white noise, thus normally distributed, the wavelet amplitude spectrum $\left|W\left(\phi_{i}, t, s\right)\right|$ of $\tilde{I}_{L y \alpha}\left(\phi_{i}, t\right)$ would be $\chi_{1}^{2}$ distributed. The $95 \%$ confidence contour lines are then determined by multiplying the background spectrum by the 95th percentile value of $\chi_{1}^{2}$. Thus the thick contours shown in Figure $3 \mathrm{~b}$ delimit regions where the wavelet amplitude spectrum is significant. The results are valid above the cross-hatched area in Figure $3 b$, which represents the Cone Of Influence (COI), where the edge effects, due to finite-length of the time series, are significant in the spectrum. The shorter period features above the boundary of the COI are truly due to non-stationary periodic signals present in the data-set.

The wavelet analysis clearly reveals the existence of significant intensity fluctuations with enhanced amplitude and periodicity within $0.5-1 \mathrm{~h}$, above the cross-hatched region, confined in a limited time interval between 14:42 UT and 15:30 UT during the CME event detected on 24 December 2006 (Figure 3b). This fluctuation is just in coincidence with the first sharp peak of the HI Ly $\alpha$ emission (black curve of Figure 3a). It is excluded that the identified feature is artificially introduced by the wavelet transform itself in presence of the abrupt jump at 15:14 UT, since this has been removed during the filtering process (red curve in Figure 3a). Hence, a real non-stationary oscillatory signal, significant at the $95 \%$ level, is found in correspondence to the transit of the CME front across the UVCS field of view.

In order to single out the most significant feature occurring at the time of the first peak in the HI Ly $\alpha$ intensity, the amplitude spectrum is averaged over the interval from $t_{1}=14: 42$ UT to $t_{2}=15: 30$ UT on 24 December 2006 (Figure 3c).

$$
\left|\bar{W}\left(\phi_{i}, s\right)\right|=\frac{\delta t}{t_{2}-t_{1}} \sum_{t=t 1}^{t 2}\left|W\left(\phi_{i}, t, s\right)\right| .
$$

This quantity, which approximates the results obtained with the Fourier spectrum of the $\tilde{I}_{L y \alpha}\left(\phi_{34}=201^{\circ}, t_{1}<t<t_{2}\right)$ series [27], clearly shows where the signal is above the $95 \%$ confidence level (dotted red line in Figure 3c), thus confirming that non-stationary oscillations with higher amplitude are found in the $\sim 0.5-1 \mathrm{~h}$ periodicity band.

Furthermore, in order to examine how the observed non-stationary fluctuations evolve during the $\mathrm{CME}$, the average wavelet amplitude can be defined as the weighted sum of the spectrum over all periodicities between 0.5 and $1 \mathrm{~h}$ (Figure $3 \mathrm{~d}$ ):

$$
\left|\bar{W}\left(\phi_{i}, t\right)\right|=\frac{\delta j \delta t}{C_{\delta}} \sum_{j=j_{1}}^{j_{2}} \frac{\left|W\left(\phi_{i}, t, s_{j}\right)\right|}{s_{j}},
$$

where $\delta j=0.125$ is the spacing between the discrete scales defined in the present analysis as fractional powers of two, $s_{j}=s_{0} 2^{j \delta j}$ ( $s_{0}$ is the Nyquist-Shannon scale), and $C_{\delta}=1.132$ is the reconstruction factor for the Paul wavelet function. 
The presence of significant fluctuations is evident at the time of the first peak in the HI Ly $\alpha$ intensity. Therefore all analysis approaches illustrated in Figure $3 b-d$ confirm that a significant non-stationary oscillatory signal exists over periodicities of $0.5-1 \mathrm{~h}$ in the interval from 14:42 UT to 15:30 UT on 24 December 2006.

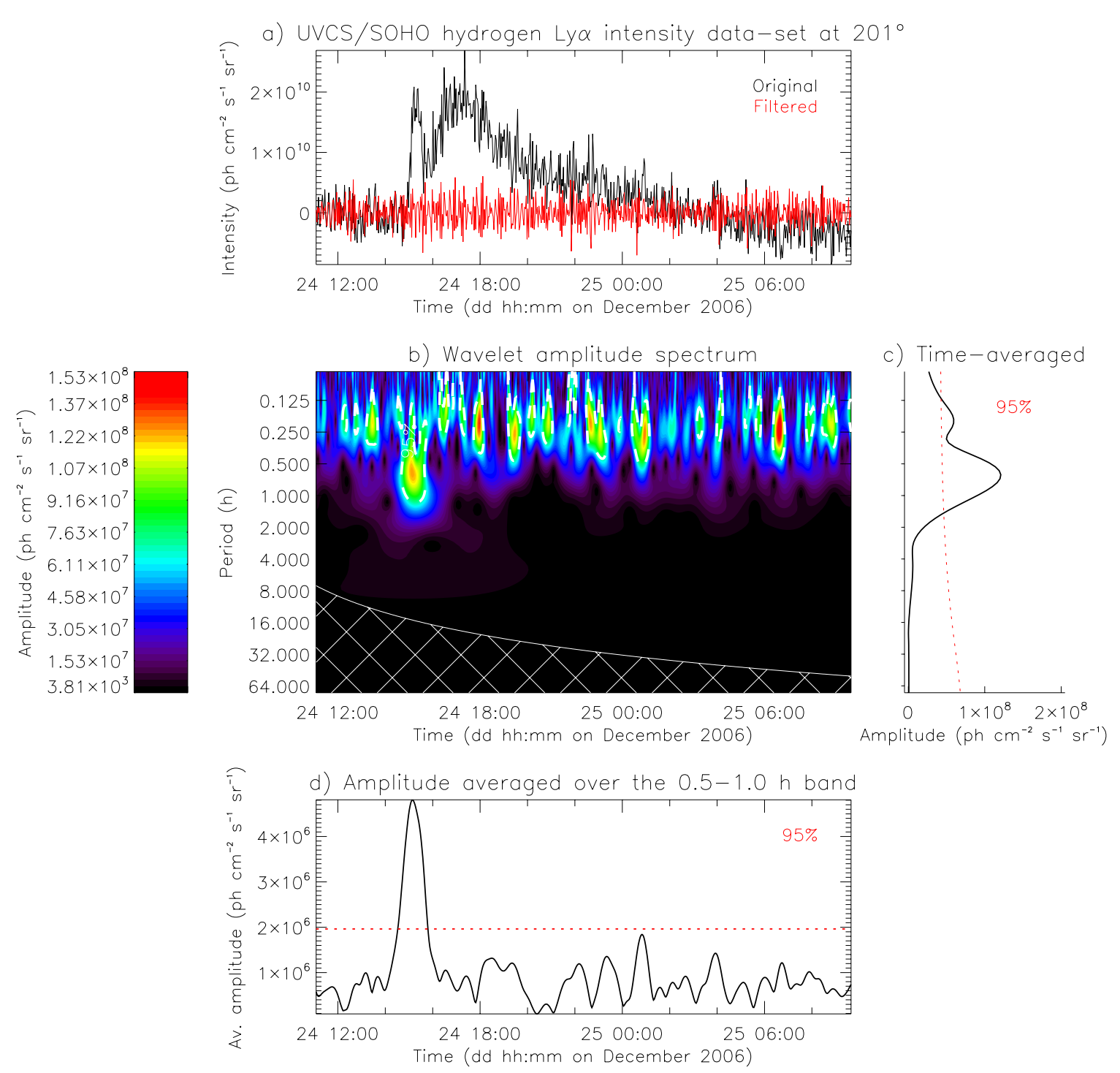

Figure 3. (a) HI Ly $\alpha$ intensity detected in pixel 34 of the UVCS slit, i.e., at $201^{\circ}$ counterclockwise from the North pole, $I_{L y \alpha}\left(\phi_{34}=201^{\circ}, t\right)$ (black curve); the time series used for the wavelet analysis, $\tilde{I}_{L y \alpha}\left(\phi_{34}=201^{\circ}, t\right)$ (red curve), has been obtained by filtering out any trend of the CME signal due to the CME transit, from $I_{L y \alpha}\left(\phi_{34}=201^{\circ}, t\right)$. (b) Wavelet amplitude spectrum of the filtered time series $\tilde{I}_{L y \alpha}\left(\phi_{34}=201^{\circ}, t\right)(\mathbf{a})$, using the Paul wavelet function; the left axis is the Fourier period (h); the bottom axis is time; the cross-hatched area marks the Cone Of Influence (COI) where edge effects become important; the thick dashed contours surround areas of statistical confidence larger than 95\%. (c) Wavelet amplitude spectrum (b) averaged over the time interval from $t_{1}=14: 42$ UT to $t_{2}=15: 30$ $\mathrm{UT},\left|\bar{W}\left(\phi_{34}=201^{\circ}, s\right)\right|$; the dotted red line is the $95 \%$ statistical confidence for a white-noise process. (d) Average of the wavelet amplitude (b) over the $0.5-1 \mathrm{~h}$ periodicity band, $\left|\bar{W}\left(\phi_{34}=201^{\circ}, t\right)\right|$; the dotted red line is the $95 \%$ confidence level. 


\subsection{Amplitude Hovmöller}

The average wavelet amplitudes, $\left|\bar{W}\left(\phi_{i}, t\right)\right|$ (Equation (4)), calculated at each heliographic latitude $\phi_{i}$, are combined into a two-dimensional contour plot, the Hovmöller plot, illustrating the dependence of the fluctuation amplitude on heliolatitude in the range from $170.5^{\circ}$ to $212.5^{\circ}$ and its evolution on time during the event. As shown by the Hovmöller plot, the wavelet amplitude of the HI Ly $\alpha$ intensity, averaged over the periodicity band of $0.5-1 \mathrm{~h}$, peaks at latitudes ranging from $200^{\circ}$ to $207^{\circ}$, although significant non-stationary oscillations are present along the entire FOV of UVCS, from $170.5^{\circ}$ to $212.5^{\circ}$ (Figure $4 \mathrm{a}$ ). In the figure, the $\mathrm{x}$-axis represents the latitude range covered by the UVCS field of view and the $y$-axis the time of observation.

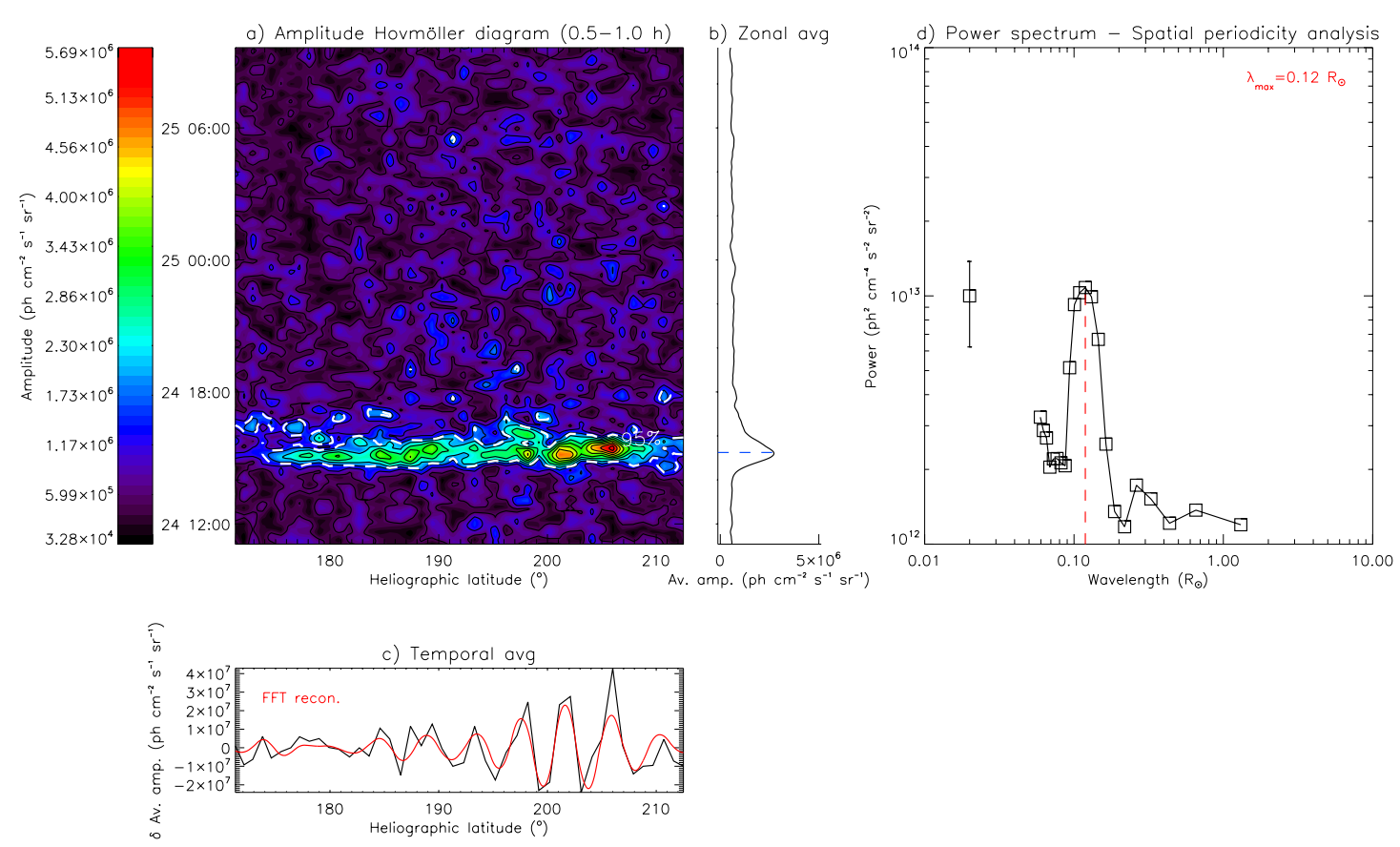

Figure 4. (a) Hovmöller amplitude diagram of the wavelet amplitude of the HI Ly $\alpha$ intensity average over the periodicity band of $0.5-1 \mathrm{~h},\left|\bar{W}\left(\phi_{i}, t\right)\right|$ (Equation (4)) at each latitude; the left axis is time; the bottom axis is heliographic latitude; the thick dashed contour is the $95 \%$ confidence level, using the corresponding white-noise background spectrum at each latitude. (b) Average of (a) over all latitudes; the blue dashed line indicates the time (15:14 UT) of the first peak in the HI Ly $\alpha$ intensity. (c) Average of (a) over the time interval from $t_{1}=14: 42$ UT to $t_{2}=15: 30$ UT on 24 December 2006, filtered out with a 6-point running average to remove the latitude dependence at large scales; the red curve is the Fourier reconstruction of the time series obtained with the dominant spatial mode inferred from the Fourier power spectrum of (c). (d) Fourier power spectrum of (c): the dominant spatial scale of $0.12 \mathrm{R}_{\odot}$ is indicated by a red dashed line.

The zonal average of the Hovmöller amplitude shows that the oscillations in the entire latitude range covered by the UVCS slit, $|\overline{\bar{W}}(t)|$, peaks in coincidence with the first enhancement in the HI Ly $\alpha$ intensity at 15:14 UT at the passage of the CME front (Figure $4 b$ ).

In order to investigate whether the oscillatory phenomena show a spatial structure, a Fast Fourier Transform (FFT) has been applied to the average of the Hovmöller amplitude over the time interval from $t_{1}=14: 42 \mathrm{UT}$ to $t_{2}=15: 30 \mathrm{UT}, \overline{|\bar{W}(\phi)|}$, when the oscillations are enhanced. As in the temporal analysis, large-scale trends are filtered out. The effect on $\mid \overline{\bar{W}(\phi) \mid}$, due to the large-scale structure of the HI Ly $\alpha$ emission (dashed line in Figure 2c), is removed by means of a 6-point moving average filter, 
to highlight fluctuations at smaller scales. The filtered time-average of the Hovmöller amplitude is shown as a black curve in Figure $4 \mathrm{c}$ as a function of latitude.

Evidence for the existence of a spatial periodicity in the oscillations observed in the latitude range where the CME is detected with UVCS, is given by a Fourier analysis. The power spectrum of the filtered latitudinal distribution of amplitude, $\overline{|\bar{W}(\phi)|}$, (Figure $4 \mathrm{~d}$ ) shows a single peak at a spatial periodicity of about $0.12 \mathrm{R}_{\odot}$. The error bar represents the $95 \%$ confidence level, derived from a 2 degree of freedom $\chi^{2}$ distribution [28]. The power of the peak at $0.12 \mathrm{R}_{\odot}$ is largely exceeding the background-level of $10^{12} \mathrm{ph}^{2} \mathrm{~cm}^{-4} \mathrm{~s}^{-2} \mathrm{sr}^{-2}$ and the error bar. The red curve in Figure $4 \mathrm{~d}$ shows the Fourier reconstruction of the filtered $\overline{|\bar{W}(\phi)|}$ spatial series, obtained by deconvolving the power spectrum on the basis of the most significant spatial periodicity, i.e., $0.12 \mathrm{R}_{\odot}$. As clearly shown in the figure, the reconstructed spatial series reproduces fairly well the original one, thus confirming the presence of a spatial periodicity of the amplitudes of the non-stationary oscillations.

\subsection{Wavelet Coherence}

The oscillatory phenomena observed along the slit are also tested for coherence by means of a wavelet coherence analysis. Given two time series at neighboring pixels, $\tilde{I}_{L y \alpha}\left(\phi_{i}, t\right)$ and $\tilde{I}_{L y \alpha}\left(\phi_{i+1}, t\right)$, with wavelet transforms $W\left(\phi_{i}, t, s\right)$ and $W\left(\phi_{i+1}, t, s\right)$, the wavelet coherence $C\left(\phi_{i} \phi_{i+1}, t, s\right)$ of the two data-sets is defined as the square of the cross-wavelet power spectrum normalized by the individual spectra

$$
C\left(\phi_{i} \phi_{i+1}, t, s\right)=\frac{\left|W\left(\phi_{i} \phi_{i+1}, t, s\right)\right|^{2}}{\left|W\left(\phi_{i}, t, s\right)\right|^{2}\left|W\left(\phi_{i+1}, t, s\right)\right|^{2}},
$$

where

$$
W\left(\phi_{i} \phi_{i+1}, t, s\right)=W\left(\phi_{i}, t, s\right) W^{*}\left(\phi_{i+1}, t, s\right)
$$

is the cross-wavelet spectrum of the two time series, with the asterisk indicating the complex conjugate. Equation (5) expresses the cross-correlation between the two time series as a function of time and frequency.

The average of $C\left(\phi_{i} \phi_{i+1}, t, s\right)$ over all periodicities between 0.5 and $1 \mathrm{~h}, \bar{C}\left(\phi_{i} \phi_{i+1}, t\right)$, yields the degree of coherence between adjacent oscillation patterns in the $0.5-1 \mathrm{~h}$ band and its temporal evolution. Figure 5a shows the diagram of the degree of coherence, or coherence index, in time and latitude. The abscissa represents the latitude between $170.5^{\circ}$ and $212.5^{\circ}$, the ordinate the time of the observation. The thick dashed contour line in Figure $5 \mathrm{a}$ is the $95 \%$ confidence level; values lying above it, have, at most, a 5\% likelihood of being due to white noise [20]. The zonal and temporal averages of the coherence index provide information on the temporal evolution and spatial distribution of the most significant coherent features (Figure $5 b, c$ ).

The quasi periodic oscillations observed in the range between 0.5 and $1 \mathrm{~h}$ show a high degree of coherence ( $\sim .8$ ) along the entire observed region (Figure $5 \mathrm{a}, \mathrm{c}$ ), with a peak in correspondence to the first peak of the Ly $\alpha$ intensity (blue dashed line in Figure 5b).

On the basis of the results achieved by means of the wavelet analysis, significant non-stationary oscillations with periods between 0.5 and $1 \mathrm{~h}$ are found in the solar corona at an average heliodistance of $1.87 \mathrm{R}_{\odot}$ in the latitude range from $170.5^{\circ}$ to $212.5^{\circ}$, during the interval from 14:42 UT to $15: 30 \mathrm{UT}$ on 24 December 2006 when a coronal mass ejection crosses the UVCS field of view. The oscillations are highly coherent in the observed region, furthermore, they exhibit a significant spatial periodicity at $\sim 0.12 \mathrm{R}_{\odot}$. 

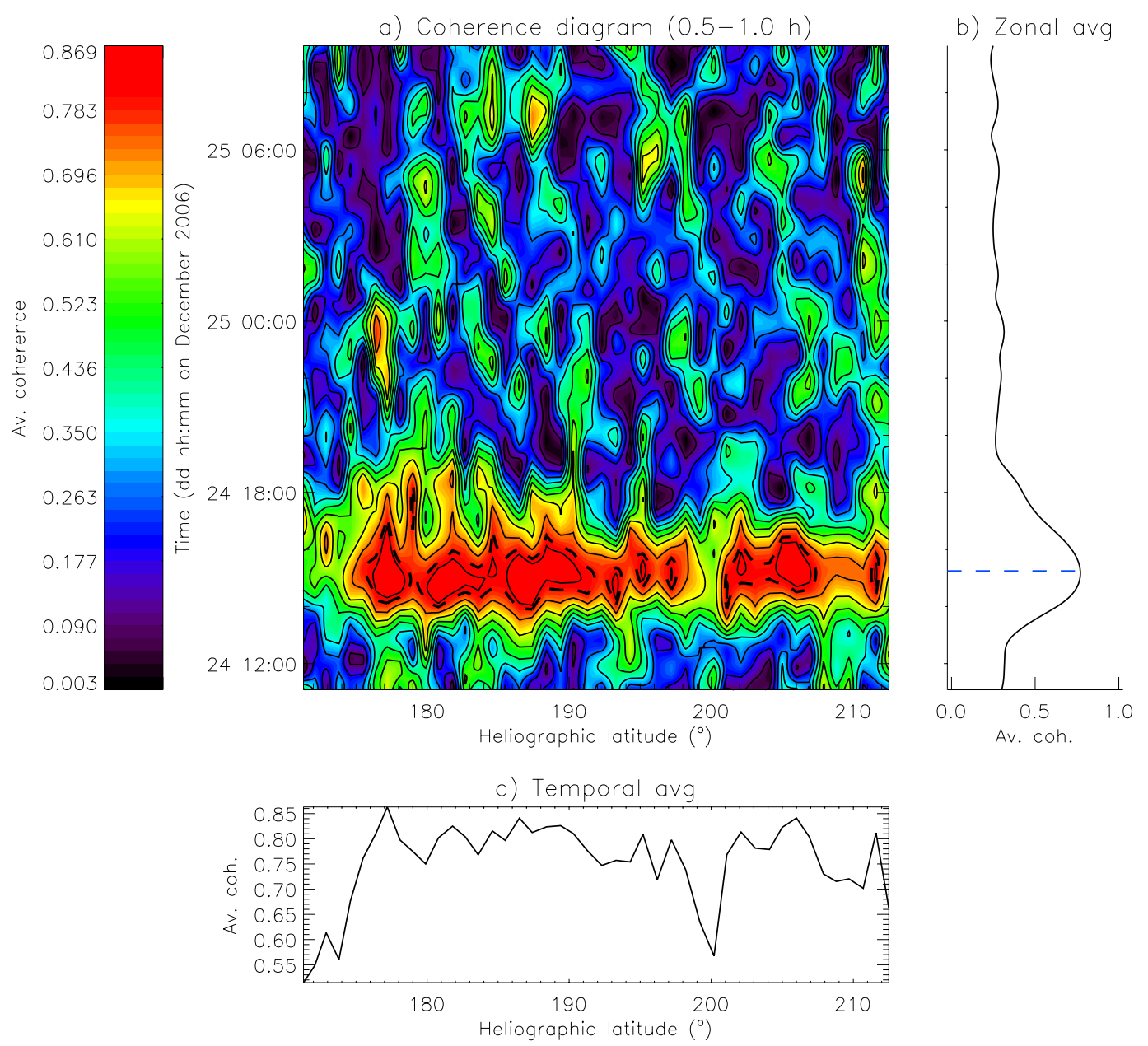

Figure 5. (a) Time-latitude diagram of the degree of coherence of the non-stationary oscillations with periods of $0.5-1 \mathrm{~h}$ along the UVCS slit; the left axis is time; the bottom axis is heliographic latitude; the thick dashed contour is the $95 \%$ confidence level. (b) Average of (a) over all latitudes; the blue dashed line indicates the time (15:14 UT) of the first peak in the HI Ly $\alpha$ intensity. (c) Average of (a) over the time interval from $t_{1}=14: 42$ UT to $t_{2}=15: 30$ UT on 24 December 2006.

\section{Discussion}

The HI Ly $\alpha$ intensity oscillations found in the analysis can be ascribed to oscillations of the density of the emitting coronal plasma. The HI Ly $\alpha$ line is produced by resonance scattering of the chromospheric Ly $\alpha$ emission from the neutral hydrogen in the corona, and its intensity $I_{L y \alpha}$ is related to the electron density $n_{e}$ and the outflow velocity of the coronal plasma $v_{\text {out }}$ via Doppler dimming effect, see [29], $I_{L y \alpha}=f\left(n_{e}, v_{\text {out }}\right)$. However, the intensity of the Ly $\alpha$ line is about 3 times more sensitive to temporal variations of the electron density $\Delta n_{e}$ than to those of the outflow velocity $\Delta v_{\text {out }}$, i.e., $\Delta I_{L y \alpha} \approx f\left(\Delta n_{e}\right)$. Hence the oscillations of the Ly $\alpha$ emission revealed by the wavelet analysis can be predominantly associated with plasma density fluctuations see also [23,29].

The results of the periodicity analysis presented in $\S 3$, clearly indicate that the non-stationary oscillations of the plasma density are associated with the passage of the CME front in the observed coronal region. When crossing the ambient corona the CME induces in the ambient plasma strong density oscillations with periodicities within 0.5 and $1 \mathrm{~h}$, with amplitudes significantly above the background level, given by the white-noise oscillations of the quiet coronal plasma. Possible effects due the transit of the CME core are instead not distinguishable from the background density oscillations 
(Figure 3d). This difference in the effects produced by the front and the core of the CME is clearly illustrated in the Hovmöller amplitude plot (Figure 4a).

The excitation of non-stationary oscillations imply that the CME at its front transfers energy and momentum to the ambient plasma and drives the generation of plasma oscillations, detectable in the UV spectrum as intensity fluctuations of the HI Ly $\alpha$ line. These oscillations are non-stationary with a lifetime of about $1-2 \mathrm{~h}$ (Figure $4 \mathrm{~b}$ ), consistent with the time of transit of the CME front across the UVCS slit. This fact suggests a confinement of the plasma oscillations at the moving front thus explaining the non-stationary nature of the oscillations. The process generating the non-stationary oscillations depends on the plasma properties along the front itself, since the oscillation amplitude is enhanced when the local density is higher (Figures $2 \mathrm{c}$ and $4 \mathrm{c}$ ).

The oscillations exhibit a well defined periodic structure along the front, as shown by the Fourier analysis performed on the spatial distribution of the amplitude of the density fluctuations (Figure 4d). The scale of the spatial periodicity is about $0.12 \mathrm{R}_{\odot}=84 \mathrm{Mm}$.

Finally, the results of the coherence analysis $(\S 3.3)$ reveal that the plasma oscillations driven by the transit of the front are coherent in phase at a high degree. That is, the plasma density is oscillating with the same phase along the interaction region between the front of the CME and the quiet solar corona (Figure 5a). These observations point to a magnetohydrodynamic process able to generate compressive structures characterized by the same phase. The degree of coherence of the plasma fluctuations is approximately constant ( 0.8) along the front of the CME (Figure $5 \mathrm{c}$ ). This implies that the oscillations are generated simultaneously by the same mechanism along the front of the mass ejection.

To summarize, the front of the CME in its interaction with the unperturbed solar corona induces the formation of coherent non-stationary density oscillations, which are generated all along the front, with a significant temporal and spatial periodicity of about $0.5-1 \mathrm{~h}$ and $84 \mathrm{Mm} \simeq 0.12 \mathrm{R}_{\odot}$, respectively.

\section{Interpretation}

The properties of the density oscillations induced in the ambient solar corona by the transit of the $\mathrm{CME}$, suggest that this phenomenon can be ascribed to the onset of the magnetic Rayleigh-Taylor (RT) instability. Indeed, the oscillations (i) originate where the front, heavier than the ambient, is decelerating into the less dense coronal plasma, (ii) are characterized by both temporal and spatial periodic pattern, and (iii) show a high degree of phase coherence.

The RT instability, one of the most important processes in astrophysical plasma, due to the interpenetration of materials, arises at the boundary layer separating a light fluid pushing on a heavier one. In this dynamic process the two fluids tend to reduce their combined potential energy. This density stratification-driven instability leads to the formation of coherent density structures, in form of fingers of lighter fluid penetrating the heavier fluid, known as 'bubbles', and 'spikes' of heavier fluid moving into the lighter fluid. The initial perturbation grows exponentially in time with a characteristic rate, which is function of the plasma properties and the physical conditions present at the discontinuity layer. In this early linear stage, the interfacial oscillations are of a single dominant wavelength. With time, the bubbles and spikes, which are initially distinct, evolve by growing, splitting, merging and rolling up into vortices which are due to the onset of secondary Kelvin-Helmholtz instabilities. In this second nonlinear stage, the plasma instabilities include a superposition of many modes (i.e., a spectrum). The interactions between modes result in a turbulent flow, characterized by a high level of mixing between the fluids and self-similarity.

The magnetic RT instability is known to be an important driver behind many observed astrophysical phenomena, such as the accretion onto magnetized compact objects e.g., [30], the generation of buoyant bubbles by radio jets in cluster of galaxies e.g., [31], and the formation of filamentary structures by pulsar winds sweeping up stellar ejecta in supernova remnants e.g., [32]. In solar physics, the magnetic RT instability is expected to be important in the emergence of magnetic flux from the solar interior and the formation of flux tubes [33], and in dark upflows propagating from underdense bubbles through quiescent prominences [34]. Recently, the RT instability has been invoked 
to explain the morphological and dynamic structure of plasma blobs falling back toward the solar surface, after the filament eruption of 7 June $2011[35,36]$

Statistical studies demonstrate that CMEs first undergo a strong acceleration phase in the lower corona, often followed by a slow deceleration during the propagation phase in the intermediate-outer corona, and that the relative duration of these two phases is strictly related to the duration of the flare when associated with the event [37-41]. The deceleration phase is related to the interaction between the ambient corona and the CME, resulting in an effective drag slowing the CME down to the speed of the local solar wind [42]. Hence, the conditions are such that the RT instability can indeed be excited at the CME front in two different locations, depending on the dynamics of the CME, as schematically shown in Figure 6. If the CME is in the acceleration phase (left panel of Figure 6) the lighter plasma in the CME cavity (likely the upper limit of the CME flux rope) is pushing the overlying denser plasma of the CME front, thus constituting a RT-unstable situation: RT plasma instabilities will be generated at the interface between the cavity and the front. Alternatively, if the CME is in the deceleration phase, as the 24 December 2006 event when crossing the observed FOV (Figure 1f), the denser plasma of the CME front is slowing down into the lighter plasma of the external corona overlying the CME (right panel of Figure 6). The interface between the front and the surrounding ambient corona is thus RT-unstable and the existence of the typical RT coherent fingers-shaped features can be inferred from the observations of coherent oscillations of the plasma density.

\section{Acceleration phase}

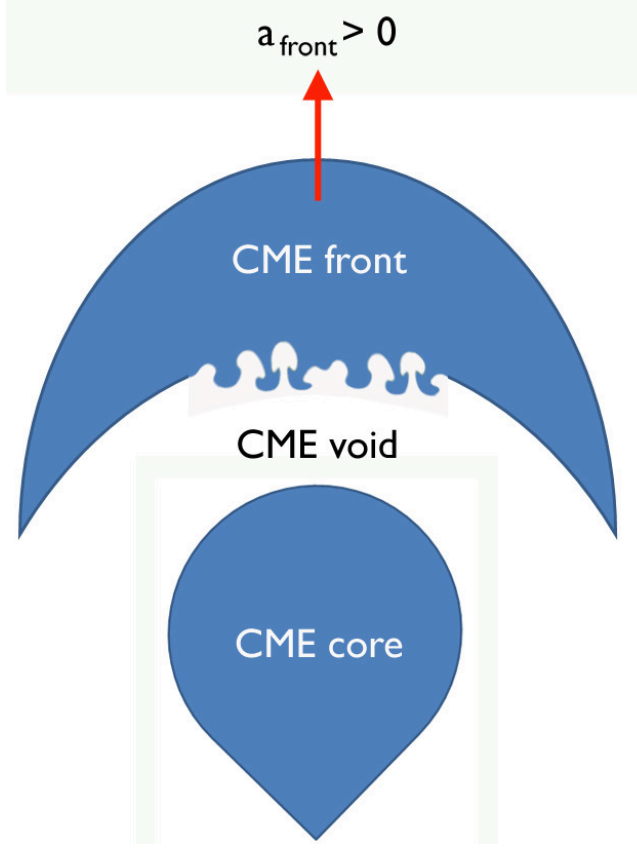

(a)

\section{Deceleration phase}

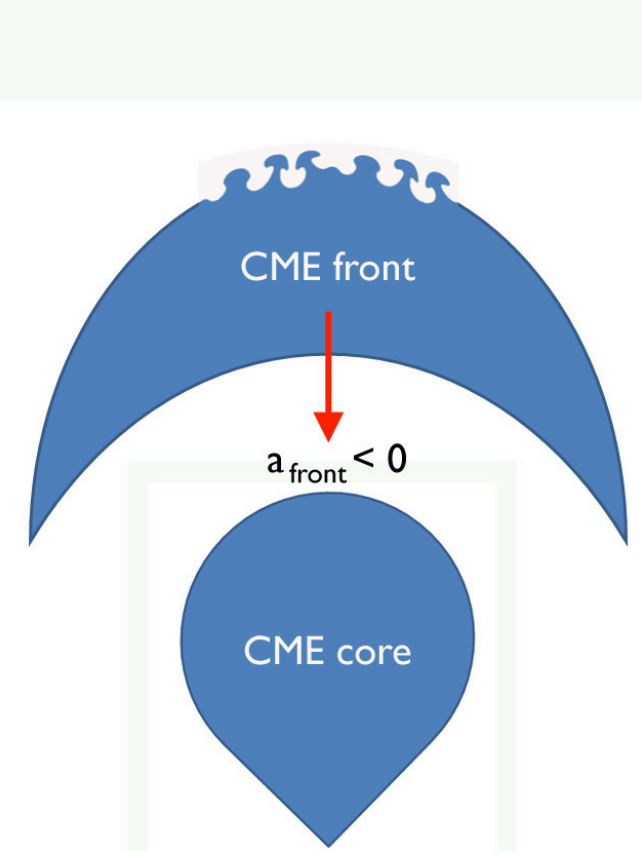

(b)

Figure 6. Two possible locations for the generation of RT plasma instabilities at the CME front during the acceleration (a) and the deceleration (b) phases (see text).

The morphology exhibited by the CME during its evolution, as displayed in the LASCO C2 white-light wavelet-filtered images of Figure 7, provides further clues to the likely onset of the magnetic RT instability at the CME front. Due to the low spatial resolution of the coronagraph (11.9" per pixel) it is difficult to distinguish, along the CME front, the filamentary features specific to the RT instability. Nevertheless, the trailing edge of the CME does not appear as the typical straight arch-shaped front, rather it seems uneven and characterized by corrugations, which dynamically 
develop during the CME expansion into ripple-shaped structures, leading to a more and more jagged front. It is however worth noting that at these heights, the magnetic RT instability is likely to be observed in its second nonlinear phase, when turbulent motions begin to develop along the CME interaction region, thus lacking of evident finger-shaped structures, broadening the interface and reducing the magnitude of the shear (Figure 7). On the other hand, at the lower heliocentric distances of the UVCS slit position (below the FOV of LASCO C2), the magnetic RT instability is still in its early linear phase (as inferred in the following on the basis of the spectral characteristics of the density fluctuations detected with UVCS): this would explain, having in mind also the different morphology exhibited by the RT instability in white light (which depends only on the plasma density) and UV (which depends also on the temperature and outflow velocity of the plasma), why UVCS is able to clearly resolve the spatial and temporal oscillations associated to the RT instability, in spite of the even lower resolution of UVCS with respect to LASCO.

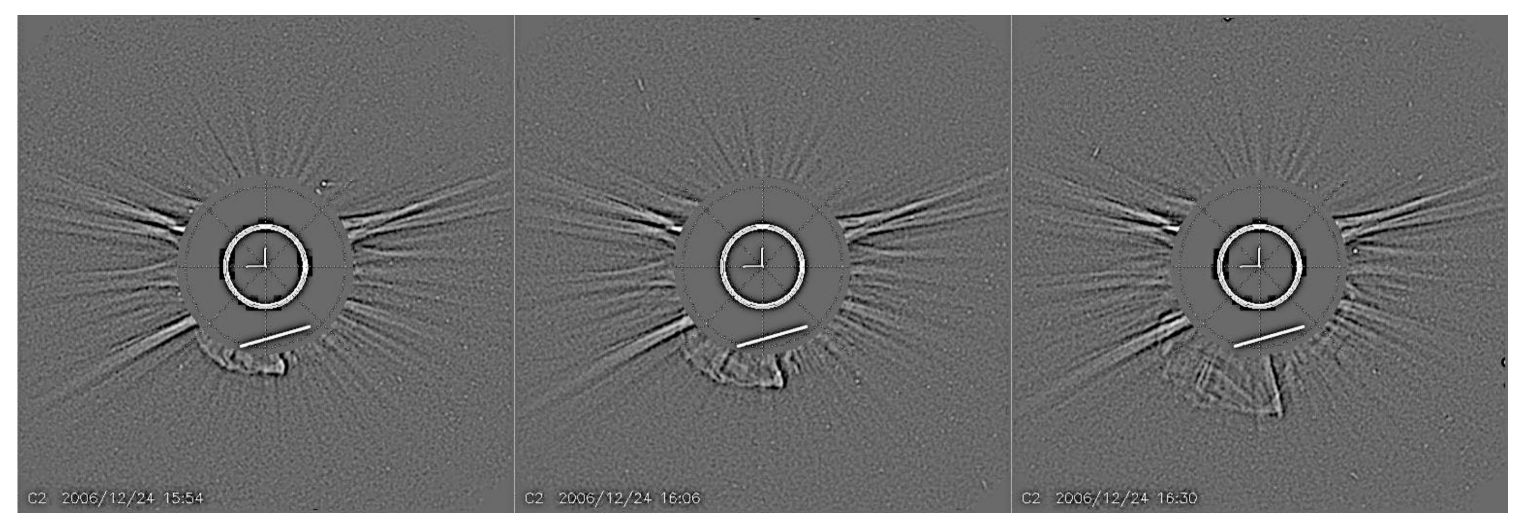

Figure 7. White light corona snapshots (wavelet filtered) acquired by LASCO C2 at 15:54, 16:06 and 16:30 UT on 24 December 2006, showing the evolution of the jagged and uneven front of the CME; the solar disk and the UVCS slit are also plotted.

It turns out that the strong and coherent density fluctuations observed along the CME front in the event under study can be interpreted as evidence for the presence RT instability in the outer solar corona. This interpretation is supported by a magnetohydrodynamic linear analysis of the RT instability, as discussed below.

The physical properties of this instability are well known. The growth rate $(\gamma)$ of the magnetic RT instability is

$$
\gamma^{2}=k^{2} g\left(\frac{A}{k}-\frac{B^{2} \cos ^{2} \theta}{2 \pi\left(\rho_{+}+\rho_{-}\right) g}\right),
$$

where $B$ is the magnetic field strength, $k$ is the wavenumber of the perturbation, $\theta$ is the angle between $B$ and the interface, $g$ is the effective gravitational acceleration at the density discontinuity, and $A$ is the Atwood number, where $A=\left(\rho_{+}-\rho_{-}\right) /\left(\rho_{+}+\rho_{-}\right)$with $\rho_{+}$the density of the heavier plasma and $\rho_{-}$the density of the lighter plasma [43]. For a magnetic field perpendicular to the interface (i.e., parallel to gravity and the density gradient in the system), Equation (7) shows that $B$ has no effect on the RT instability, which develops as in the purely hydrodynamic case. However, for a horizontal magnetic field (as expected at the density discontinuity separating the CME front and the surrounding solar corona), the magnetic tension force modifies the growth rate of the RT instability, thus regulating its evolution in the linear phase, until the RT instability can be completely suppressed.

In order to compare the physical characteristics of the plasma density oscillations observed along the CME front with the predictions of the linear stability analysis, the parameters involved in Equation (7) are estimated as follows.

The growth rate of the plasma density oscillations observed along the CME front is estimated to be of the order of $1 \mathrm{~h}^{-1}$ on the basis of the following considerations. The existence of a unique peak in the wavelet spectrum during the transit of the CME front (Figure 3c), indicates density fluctuations 
of a single wavelength. This implies that the RT instability is still developing in its linear phase. The lifetime of the plasma oscillations of about one hour (Figure $4 \mathrm{~b}$ ) has to be of the order of the linear characteristic timescale $\left(\gamma^{-1}\right)$ of the RT plasma instability. As a matter of fact, if the timescale $\gamma^{-1}$ were much smaller than the lifetime of the oscillations, which in our study is an observational parameter, the RT structures would have already decayed into a turbulent mixed flow during the front transit time. Hence, the global wavelet spectrum would not exhibit a unique periodicity, but a continuous spectrum of modes. On the other hand, if the linear characteristic timescale $\gamma^{-1}$ were much larger than the oscillation lifetime, the plasma instability would not be well-developed yet, thus the density fluctuations due to the RT instability would be indistinguishable from the background noise. In addition, also the width of the peak observed in the global wavelet spectrum (Figure 3c) can provide further information on the instability growth rate. The power spectrum of a sinusoidal perturbation, $\eta(t)=\eta_{0} e^{i \omega t}$, is characterized by a sharp peak at the frequency $v=\frac{2 \pi}{\omega}$, but if the amplitude of the sinusoidal perturbation grows exponentially with a rate $\gamma, \eta(t)=\eta_{0} e^{\gamma t} e^{i \omega t}$, the peak at the frequency $v$ in the power spectrum becomes wider and wider as the linear formation timescale $\gamma^{-1}$ grows. The width of the peak in the observed global wavelet spectrum is consistent with a formation timescale ranging from $0.5 \mathrm{~h}$ up to $1.5 \mathrm{~h}$. These values indeed agree with the observed lifetime of the plasma oscillations (Figure $4 \mathrm{~b}$ ), thus this is an estimate of the linear characteristic timescale of the RT instabilities, i.e., $\gamma^{-1} \sim 1 \mathrm{~h}$.

The plasma densities of the heavier CME front, $\rho_{+}$, and of the overlying more rarefied unperturbed solar corona, $\rho_{-}$, where the CME is expanding, can be derived from the intensity of the HI Ly $\alpha 1216 \AA$ line, measured before and during the transit of the CME front $\left(I_{\text {corona }}=2.8 \times 10^{10} \mathrm{ph} \mathrm{cm}^{-2} \mathrm{~s}^{-1} \mathrm{sr}^{-1}\right.$ and $I_{\text {front }}=1.0 \times 10^{10} \mathrm{ph} \mathrm{cm}^{-2} \mathrm{~s}^{-1} \mathrm{sr}^{-1}$, respectively). The HI Ly $\alpha$ emission is due to the scattering of chromospheric photons by hydrogen atoms in the corona, thus the intensity is given by the relation:

$$
I_{L y \alpha}=\frac{1}{4 \pi} b h v_{0} B_{12} \int_{L O S} \int_{\Omega} p(\varphi) \mathrm{d} \omega F\left(w_{n}\right) n_{H} \mathrm{~d} l,
$$

where $b$ is the branching ratio for radiative de-excitation, $v_{0}$ is the reference frequency, $B_{12}$ is the Einstein coefficient for absorption [44], $n_{H}$ is the number density of the hydrogen atoms and

$$
F\left(w_{n}\right)=\int_{-\infty}^{+\infty} I_{\text {exc }}\left[\left(v_{0}+\delta v\left(v_{n}\right)\right), \mathbf{n}\right] f_{n}\left(v_{n}-w_{n}\right) \mathrm{d} v_{n}
$$

represents the Doppler dimming introduced by the radial expansion of the coronal plasma [45,46], where $I_{\text {exc }}\left(v_{0}+\delta v, \mathbf{n}\right)$ is the intensity of the exciting spectrum, emitted from the lower atmosphere along the direction of the incident radiation $\mathbf{n}$. The frequency $\left(v_{0}+\delta v\right)$ at which the absorption occurs, depends on the velocity $v_{n}$ of the absorbing ions along the direction of the incident radiation and the quantity $\delta v=v_{0}(\mathbf{v} \cdot \mathbf{n}) / c$ is the Doppler shift of the exciting radiation in the frame of reference of the scattering ion. The distribution function of the velocity of the absorbing ions along the direction $\mathbf{n}$, $f_{n}\left(v_{n}-w_{n}\right)$, is assumed to be Maxwellian. The vector $\mathbf{w}$ represents the mean velocity of the coronal ions in the point of scattering, which corresponds to the velocity of the expansion of the solar corona. The projection of this vector along the direction of the incident radiation is given by $w_{n}=\mathbf{w} \cdot \mathbf{n}$. The function $p(\varphi)$ takes into account the geometry of the scattering process [46], $\varphi$ is the angle between $\mathbf{n}$ and the LOS, $\mathrm{d} \omega$ is the infinitesimal solid angle around $\mathbf{n}$, and $\Omega$ is the solid angle subtended by the solar disk at the point of scattering.

During the solar activity minimum, the speed of the outflowing plasma in a polar coronal hole at the UVCS position is of the order of $v_{\text {corona }} \sim 190 \mathrm{~km} \mathrm{~s}^{-1}$ e.g., [29]. The expansion speed of the CME front when crossing the UVCS slit, inferred from the height-time plot of Figure $1 \mathrm{f}$, is $v_{\text {front }} \sim 268 \mathrm{~km} \mathrm{~s}^{-1}$ (Figure 1f). At the poles the most significant contribution to the HI Ly $\alpha$ line intensity is given by the emission near the plane of the sky, because of the rapid decrease of density with increasing heliocentric distance. Hence, $I_{L y \alpha} \approx \frac{1}{4 \pi} b h v_{0} B_{12} n_{H} \int_{\Omega} p(\varphi) F\left(w_{n}\right) \mathrm{d} \omega$. Since, to a first approximation, the CME front and the quiet coronal hole can be assumed to have comparable temperatures and 
elemental compositions (so that the mass densities, $\rho_{+}$and $\rho_{-}$, are related to the corresponding number densities, $n_{+}$and $n_{-}$, by the same multiplicative factor), the Atwood number $A$ can be expressed as

$$
\begin{aligned}
A \sim & \frac{I_{\text {front }} / \int_{\Omega} p(\varphi) F\left(v_{\text {front }}\right) \mathrm{d} \omega-\cdots}{I_{\text {front }} / \int_{\Omega} p(\varphi) F\left(v_{\text {front }}\right) \mathrm{d} \omega+\cdots} \\
& \frac{-I_{\text {corona }} / \int_{\Omega} p(\varphi) F\left(v_{\text {corona }}\right) \mathrm{d} \omega}{+I_{\text {corona }} / \int_{\Omega} p(\varphi) F\left(v_{\text {corona }}\right) \mathrm{d} \omega} \sim 3.45 \times 10^{-2} .
\end{aligned}
$$

It is worth noting that the Atwood number is very low, indicating that the CME front and the surrounding corona have similar densities.

The mass density of the lighter surrounding corona $\rho_{-}$for a fully ionized plasma with $10 \%$ helium is $\rho=1.18 m_{p} n_{e}=4.1 \times 10^{-19} \mathrm{~g} \mathrm{~cm}^{-3}$, where $m_{p}$ is the proton mass and $n_{e} \sim 2.1 \times 10^{5} \mathrm{~cm}^{-3}$ is the typical electron number densities measured in polar coronal holes at the height of the UVCS slit e.g., [29].

During the transit across the FOV, the CME is slowing down with an almost constant deceleration, $\sim-39 \mathrm{~m} \mathrm{~s}^{-2}$ (Figure 1f), which corresponds to the effective gravitational acceleration undergone by the denser plasma of the CME front, $g \sim 39 \mathrm{~m} \mathrm{~s}^{-2}$.

In order to validate the RT linear theory for the plasma perturbations observed at the CME front, an estimate of the strength of the magnetic field when perturbed by the transit of the CME, $B$, would be needed. There exists a growing literature on measurements of CME magnetic fields in the solar corona e.g., [47-56]. Despite the quite large variability of $B$ estimations, due to the analysis of different events with different techniques (e.g., Faraday-rotation or moving type IV bursts observations, conservation principle of magnetic helicity, determination by means of shock properties), it can be however confidently stated that the magnetic field strength $B$ associated to the trailing edge of a CME is less than $1 \mathrm{G}$ when detected at a heliocentric distance of about $2 \mathrm{R}_{\odot}$.

It turns out that the ratio between the first and the second term of Equation (7), which represents the imbalance between the hydrodynamic force and the magnetic tension, is

$$
\begin{aligned}
\frac{A}{k} \cdot \frac{2 \pi\left(\rho_{+}+\rho_{-}\right) g}{B^{2} \cos ^{2} \theta} & =A \lambda \cdot \frac{\left(\rho_{+}+\rho_{-}\right) g}{B^{2} \cos ^{2} \theta} \\
& >10^{8} A \lambda\left(\rho_{+}+\rho_{-}\right) g \\
& >2 \times 10^{8} A \lambda \rho_{-} g \sim 10
\end{aligned}
$$

having in mind that $\rho_{+}>\rho_{-}, B<1 \mathrm{G}=10^{-4} \mathrm{~T}$ and $\cos ^{2} \theta \leq 1$, and where the perturbation wavelength $\lambda=2 \pi / k$ has been assumed equal to the spatial periodicity $(84 \mathrm{Mm})$ exhibited by the non-stationary density oscillations observed with UVCS along the boundary layer separating the CME expanding into the solar corona (Section 3.2) and interpreted as due to the magnetic Rayleigh-Taylor instability.

Since the second term of Equation (7) is at least ten times lower than the first one even in the case of a magnetic field with a significant component parallel to the interface, the magnetic tension can be considered negligible with respect to the hydrodynamic force. It follows that the growth rate of the magnetic RT instability can be approximated to $\gamma^{2}=k g A$, expression valid in the hydrodynamic case.

On the basis of the parameters of the instability derived from this observational study, for a RT perturbation growing exponentially with a rate $\gamma \sim 1 \mathrm{~h}^{-1}$, at the interface of two fluids with $A \sim 3.45 \times 10^{-2}$ and under the influence of a constant acceleration $g \sim 39 \mathrm{~m} \mathrm{~s}^{-2}$, the linear theory predicts a unique instability wavelength $\lambda=2 \pi / k=2 \pi g A \gamma^{-2} \sim 110 \mathrm{Mm}=0.16 \mathrm{R}_{\odot}$. This value is consistent with the observed dominant spatial scale of about $84 \mathrm{Mm}=0.12 \mathrm{R}_{\odot}$ of the plasma oscillations (Figure $4 \mathrm{c}, \mathrm{d}$ ), therefore this is an indication for the existence of the Rayleigh-Taylor instability generated by the transit in a quiet coronal hole of a decelerating, denser CME front. This result can be considered as the first indirect evidence of the presence of the magnetic Rayleigh-Taylor instability in the low- $\beta$ plasma of the outer solar corona. 


\section{Conclusions}

This paper reports the first detection of a Rayleigh-Taylor instability in the outer solar corona, due to the transit, in a quiet southward polar coronal hole, of the front of the coronal mass ejection observed in UV on 24 December 2006. This instability is expected to be a very rare phenomenon, difficult to detect since, in order for the RT instability to be triggered, it is required that a CME is slowing down in a coronal plasma having a similar density of the CME front, two really restrictive conditions that makes the observation of the RT instability at the front of CMEs extremely difficult. The CME analyzed in this paper fulfills both requirements, allowing the investigation of the RT instability at the interface separating the CME front and the surrounding corona, by means of spectral analyses. The RT coherent oscillations develop on timescales of about one hour all along the front although their amplitude is enhanced where the density of the front is higher. They are characterized by a dominant temporal and spatial periodicity of $\sim 0.5-1 \mathrm{~h}$ and $\sim 84 \mathrm{Mm}=0.12 \mathrm{R}_{\odot}$, respectively. The growth rate $\left(\gamma \sim 1 \mathrm{~h}^{-1}\right)$ and the physical parameters $\left(A \sim 3.45 \times 10^{-2}, g \sim 39 \mathrm{~m} \mathrm{~s}^{-2}, \lambda=2 \pi / k=84 \mathrm{Mm}\right)$ of the RT instability are consistent with the values expected in the linear phase of the instability evolution.

Application of the wavelet analysis technique to UVCS observations of coronal mass ejections might lead to significant progress toward a full description and characterization of the interaction between the CMEs and the solar corona. This approach might also allow the assessment of still unknown physical quantities, by measuring the amplitude and the properties of the RT plasma perturbations induced by the transit of the CME front. As an example, the unknown strength of the coronal magnetic field perturbed by the passage of the magnetized plasma carried out by the CME, can be constrained on the basis of the linear analysis of the RT instability. The growth rate definition by linear stability theory given in Equation (7) implies that the quantity within parentheses must be positive. It turns out that for the idealized case of two inviscid, perfectly conducting fluids separated by a contact discontinuity with an uniform magnetic field $B$ parallel to the interface undergoing constant acceleration $g$, the linear analysis demonstrates that for modes parallel to the magnetic field there exists a critical magnetic field $B_{c}=\left[2 \pi\left(\rho_{+}-\rho_{-}\right) A g / k\right]^{1 / 2}$, above which the RT instability is completely suppressed. The observational evidence for RT plasma instabilities along the front of the CME implies that $B<B_{c} \lesssim 3 \mathrm{G}$, thus allowing the estimation of an upper limit for the strength of the coronal magnetic field perturbed by the transit of a coronal mass ejection.

The investigation of the plasma oscillations due to the RT instability driven by the CME expansion might also provide information on the energetics of the CME. As a matter of fact, during the nonlinear phase of the instability, the RT fingers are unstable to secondary Kelvin-Helmholtz (KH) instability, because of the differential shearing motion across the interface between the penetrating material associated to the RT structures and the unperturbed solar corona ahead the front of the CME. At a later stage of evolution relative to the one observed in this study, this flow-driven instability leads to the formation of large, coherent vortices between the fluids due to the roll-up of the shear flow. Since the magnetic field lines are frozen into the fluid, they are stretched by the vortical motions into an anti-parallel geometry. As the magnetic field lines are folded and twisted by the vortical fluid motion, kinetic energy of the KH vortices is transferred to magnetic energy. This process continues until the wrapped magnetic field lines begin to reconnect, thus dissipating magnetic into thermal energy e.g., [57,58], leading to significant heating of the CME plasma. Thus, the coupling of the RT and $\mathrm{KH}$ instabilities in the nonlinear stage might play a key role, among the other candidate physical mechanisms [59], in the heating of the coronal plasma carried out by CMEs.

In this respect, it would be very interesting to investigate the nonlinear temporal (and spatial) evolution of the RT instability during the expansion of the CME, by using an extensive set of UVCS CME-data at different heights. In fact, at larger distances the RT plasma structures should evolve towards a turbulent and mixed flow, and both the height of the interacting, say mixing, region between the two fluids and the properties of the RT instability (such as the mode composition, the growth rate and the self-similarity) should evolve in accordance with the nonlinear theory [60]. This experimental result would represent a further evidence for the presence of RT instabilities along the front of coronal 
mass ejections and might also drive to constrain some observationally unknown parameters, such as the viscous and diffusion scales, and the surface tension, which controls the evolution of the RT instability [61]. However, this is devoted to a future work.

Author Contributions: Conceptualization, D.T.; methodology, D.T.; validation, D.T. and F.C.; formal analysis, D.T. and F.C.; investigation, D.T.; data curation, A.B.; writing-original draft preparation, D.T., A.B. and E.A.; writing-review and editing, D.T.; visualization, D.T. and A.B.; supervision, E.A.

Funding: This research was funded by the Italian Space Agency grant number I/013/12/0.

Conflicts of Interest: The authors declare no conflict of interest.

\section{References}

1. Webb, D.F. Coronal mass ejections: The key to major interplanetary and geomagnetic disturbances. Rev. Geophys. 1995, 33, 577-583. [CrossRef]

2. Sheeley, N.R., Jr.; Walters, J.H.; Wang, Y.M.; Howard, R.A. Continuous tracking of coronal outflows: Two kinds of coronal mass ejections. J. Geophys. Res. Space Phys. 1999, 104, 24739-24767. [CrossRef]

3. Aschwanden, M.J.; Caspi, A.; Cohen, C.M.S.; Holman, G.; Jing, J.; Kretzschmar, M.; Kontar, E.P.; McTiernan, J.M.; Mewaldt, R.A.; O'Flannagain, A.; et al. Global Energetics of Solar Flares. V. Energy Closure in Flares and Coronal Mass Ejections. Astrophys. J. 2017, 836, 17. [CrossRef]

4. Raymond, J.C.; Thompson, B.J.; St. Cyr, O.C.; Gopalswamy, N.; Kahler, S.; Kaiser, M.; Lara, A.; Ciaravella, A.; Romoli, M.; O'Neal, R. SOHO and radio observations of a CME shock wave. Geophys. Res. Lett. 2000, 27, 1439-1442. [CrossRef]

5. Mancuso, S.; Raymond, J.C.; Kohl, J.; Ko, Y.-K.; Uzzo, M.; Wu, R. UVCS/SOHO observations of a CME-driven shock: Consequences on ion heating mechanisms behind a coronal shock. A\&A 2002, 383, 267-274. [CrossRef]

6. Korreck, K.E.; Zurbuchen, T.H.; Lepri, S.T.; Raines, J.M. Heating of Heavy Ions by Interplanetary Coronal Mass Ejection Driven Collisionless Shocks. Astrophys. J. 2007, 659, 773-779. [CrossRef]

7. Reames, D.V. Particle acceleration at the Sun and in the heliosphere. Space Sci. Rev. 1999, 90. [CrossRef]

8. Chrysaphi, N.; Kontar, E.P.; Holman, G.D.; Temmer, M. CME-driven Shock and Type II Solar Radio Burst Band Splitting. Astrophys. J. 2018, 868, 79. [CrossRef]

9. Zucca, P.; Morosan, D.E.; Rouillard, A.P.; Fallows, R.; Gallagher, P.T.; Magdalenic, J.; Klein, K.-L.; Mann, G.; Vocks, C.; Carley, E.P.; et al. Shock location and CME 3D reconstruction of a solar type II radio burst with LOFAR. A\&A 2018, 615, A89. [CrossRef]

10. Attrill, G.D.R.; Harra, L.K.; van Driel-Gesztelyi, L.; Démoulin, P. Coronal “Wave”: Magnetic Footprint of a Coronal Mass Ejection? Astrophys. J. 2007, 656, L101-L104.

11. Van Driel-Gesztelyi, P.; Démoulin, J.L.; Culhane, S.A.; Matthews, L.K.; Harra, C.H.; Mandrini, K.L.; Klein, H.; Kurokawa, C.P.G.L. A Multiple Flare Scenario where the Classic Long-Duration Flare Was Not the Source of a CME. Sol. Phys. 2007, 240. [CrossRef]

12. Bemporad, A.; Soenen, A.; Jacobs, C.; Landini, F.; Poedts, S. SIDE Magnetic Reconnections Induced by Coronal Mass Ejections: Observations and Simulations. Astrophys. J. 2010, 718, 251-265. [CrossRef]

13. Low, B.C. Coronal mass ejections, magnetic flux ropes, and solar magnetism. J. Geophys. Res. Space Phys. 2001, 106, 25141-25163. [CrossRef]

14. Foullon, C.; Verwichte, E.; Nakariakov, V.M.; Nykyri, K.; Farrugia, C.J. Magnetic kelvin-helmholtz instability at the sun. Astrophys. J. 2011, 729, L8. [CrossRef]

15. Ofman, L.; Thompson, B.J. Sdo/aia observation of kelvin-helmholtz instability in the solar corona. Astrophys. J. 2011, 734, L11. [CrossRef]

16. Domingo, V.; Fleck, B.; Poland, A.I. The SOHO Mission, 1st ed.; Springer: Dordrecht, The Netherlands, 1996.

17. Brueckner, G.E.; Howard, R.A.; Koomen, M.J.; Korendyke, C.M.; Michels, D.J.; Moses, J.D.; Socker, D.G.; Dere, K.P.; Lamy, P.L.; Llebaria, A.; et al. The Large Angle Spectroscopic Coronagraph (LASCO). Sol. Phys. 1995, 162. [CrossRef]

18. De Moortel, I.; Ireland, J.; Walsh, R. Observation of oscillations in coronal loops. A\&A 2000, 355, L23-L26.

19. Morgan, H.; Habbal, S.R.; Li, X. Hydrogen Lya Intensity Oscillations Observed by theSolar and Heliospheric ObservatoryUltraviolet Coronagraph Spectrometer. Astrophys. J. 2004, 605, 521-527. [CrossRef] 
20. Bloomfield, D.S.; McAteer, R.T.J.; Lites, B.W.; Judge, P.G.; Mathioudakis, M.; Keenan, F.P. Wavelet Phase Coherence Analysis: Application to a Quiet-Sun Magnetic Element. Astrophys. J. 2004, 617, 623-632. [CrossRef]

21. Telloni, D.; Bruno, R.; D'Amicis, R.; Pietropaolo, E.; Carbone, V. Wavelet analysis as a tool to localize magnetic and cross-helicity events in the solar wind. Astrophys. J. 2012, 751, 19. [CrossRef]

22. Telloni, D.; Perri, S.; Bruno, R.; Carbone, V.; Amicis, R.D. An analysis of magnetohydrodynamic invariants of magnetic fluctuations within interplanetary flux ropes. Astrophys. J. 2013, 776, 3. [CrossRef]

23. Bemporad, A.; Matthaeus, W.H.; Poletto, G. Low-Frequency Ly $\alpha$ Power Spectra Observed by UVCS in a Polar Coronal Hole. Astrophys. J. 2008, 677, L137-L140. [CrossRef]

24. Telloni, D.; Bruno, R.; Carbone, V.; Antonucci, E.; D'Amicis, R. Statistics of density fluctuations during the transition from the outer solar corona to the interplanetary space. Astrophys. J. 2009, 706, 238-243. [CrossRef]

25. Dolla, L.; Marqué, C.; Seaton, D.B.; Doorsselaere, T.V.; Dominique, M.; Berghmans, D.; Cabanas, C.; Groof, A.D.; Schmutz, W.; Verdini, A.; et al. Time delays in quasi-periodic pulsations observed during the x2.2 solar flare on 2011 february 15. Astrophys. J. 2012, 749, L16. [CrossRef]

26. Torrence, C.; Compo, G.P. A Practical Guide to Wavelet Analysis. Bull. Am. Meteorol. Soc. 1998, 79, 61-78. [CrossRef]

27. Percival, D.P. On estimation of the wavelet variance. Biometrika 1995, 82, 619-631. [CrossRef]

28. Spiegel, M.R. Schaum's Outline of Theory and Problems of Probability and Statistics; Mcgraw-Hill: New York, NY, USA, 1975.

29. Telloni, D.; Antonucci, E.; Bruno, R.; D'Amicis, R. Persistent and self-similar large-scale density fluctuations in the solar corona. Astrophys. J. 2009, 693, 1022-1028. [CrossRef]

30. Arons, J.; Lea, S.M. Accretion onto magnetized neutron stars - Structure and interchange instability of a model magnetosphere. Astrophys. J. 1976, 207, 914.

31. Robinson, K.; Dursi, L.J.; Ricker, P.M.; Rosner, R.; Calder, A.C.; Zingale, M.; Truran, J.W.; Linde, T.; Caceres, A.; Fryxell, B.; et al. Morphology of Rising Hydrodynamic and Magnetohydrodynamic Bubbles from Numerical Simulations. Astrophys. J. 2004, 601, 621-643. [CrossRef]

32. Hester, J.J.; Stone, J.M.; Scowen, P.A.; Jun, B.I.; John, S.I.G.; Norman, M.L.; Ballester, G.E.; Burrows, C.J.; Casertano, S.; Clarke, J.T.; et al. WFPC2 Studies of the Crab Nebula. III. Magnetic Rayleigh-Taylor Instabilities and the Origin of the Filaments. Astrophys. J. 1996, 456, 225. [CrossRef]

33. Isobe, H.; Miyagoshi, T.; Shibata, K.; Yokoyama, T. Three-Dimensional Simulation of Solar Emerging Flux Using the Earth Simulator I. Magnetic Rayleigh-Taylor Instability at the Top of the Emerging Flux as the Origin of Filamentary Structure. Publ. Astron. Soc. Jpn. 2006, 58, 423-438. [CrossRef]

34. Berger, T.E.; Slater, G.; Hurlburt, N.; Shine, R.; Tarbell, T.; Title, A.; Lites, B.W.; Okamoto, T.J.; Ichimoto, K.; Katsukawa, Y.; et al. Quiescent prominence dynamics observed with thehinodesolar optical telescope. i. turbulent upflow plumes. Astrophys. J. 2010, 716, 1288-1307. [CrossRef]

35. Innes, D.E.; Cameron, R.H.; Fletcher, L.; Inhester, B.; Solanki, S.K. Break up of returning plasma after the 7 June 2011 filament eruption by Rayleigh-Taylor instabilities. AEA 2012, 540, L10. [CrossRef]

36. Carlyle, J.; Williams, D.R.; van Driel-Gesztelyi, L.; Innes, D.; Hillier, A.; Matthews, S. Investigating the dynamics and density evolution of returning plasma blobs from the 2011 june 7 eruption. Astrophys. J. 2014, $782,87$.

37. Zhang, J.; Dere, K.P.; Howard, R.A.; Kundu, M.R.; White, S.M. On the Temporal Relationship between Coronal Mass Ejections and Flares. Astrophys. J. 2001, 559, 452-462. [CrossRef]

38. Zhang, J.; Dere, K.P.; Howard, R.A.; Vourlidas, A. A Study of the Kinematic Evolution of Coronal Mass Ejections. Astrophys. J. 2004, 604, 420-432. [CrossRef]

39. Qiu, J.; Wang, H.; Cheng, C.Z.; Gary, D.E. Magnetic Reconnection and Mass Acceleration in Flare-Coronal Mass Ejection Events. Astrophys. J. 2004, 604, 900-905. [CrossRef]

40. Temmer, M.; Veronig, A.M.; Vršnak, B.; Rybák, J.; Gömöry, P.; Stoiser, S.; Maričić, D. Acceleration in Fast Halo CMEs and Synchronized Flare HXR Bursts. Astrophys. J. 2008, 673, L95-L98. [CrossRef]

41. Jiang, B.; Chen, Y.; Wang, J.; Su, Y.; Zhou, X.; Safi-Harb, S.; DeLaney, T. Cavity of molecular gas associated with supernova remnant 3C 397. Astrophys. J. 2010, 712, 1147-1156. [CrossRef]

42. Maloney, S.A.; Gallagher, P.T. Solar wind drag and the kinematics of interplanetary coronal mass ejections. Astrophys. J. 2010, 724, L127-L132. [CrossRef]

43. Chandrasekhar, S. Hydrodynamic and Hydromagnetic Stability; Oxford University Press: Oxford, UK, 1961. 
44. Allen, C. Astrophysical Quantities; Springer: New York, NY, USA, 1973.

45. Withbroe, G.L.; Kohl, J.L.; Weiser, H.; Munro, R.H. Probing the solar wind acceleration region using spectroscopic techniques. Space Sci. Rev. 1982, 33. [CrossRef]

46. Noci, G.; Kohl, J.L.; Withbroe, G.L. Solar wind diagnostics from Doppler-enhanced scattering. Astrophys. J. 1987, 315, 706. [CrossRef]

47. Bastian, T.S.; Pick, M.; Kerdraon, A.; Maia, D.; Vourlidas, A. The Coronal Mass Ejection of 1998 April 20: Direct Imaging at Radio Wavelengths. Astrophys. J. 2001, 558, L65-L69. [CrossRef]

48. Mancuso, S.; Raymond, J.C.; Kohl, J.; Ko, Y.-K.; Uzzo, M.; Wu, R. Plasma properties above coronal active regions inferred from SOHO/UVCS and radio spectrograph observations. AEA 2003, 400, 347-353. [CrossRef]

49. Cho, K.S.; Lee, J.; Gary, D.E.; Moon, Y.J.; Park, Y.D. Magnetic Field Strength in the Solar Corona from Type II Band Splitting. Astrophys. J. 2007, 665, 799-804. [CrossRef]

50. Jensen, E.A.; Russell, C.T. Faraday rotation observations of CMEs. Geophys. Res. Lett. 2008, 35. [CrossRef]

51. Chen, Y.; Feng, S.W.; Li, B.; Song, H.Q.; Xia, L.D.; Kong, X.L.; Li, X. A coronal seismological study with streamer waves. Astrophys. J. 2011, 728, 147. [CrossRef]

52. Gopalswamy, N.; Nitta, N.; Akiyama, S.; Mäkelä, P.; Yashiro, S. Coronal magnetic field measurement from euv images made by thesolar dynamics observatory. Astrophys. J. 2011, 744, 72. [CrossRef]

53. Kwon, R.Y.; Ofman, L.; Olmedo, O.; Kramar, M.; Davila, J.M.; Thompson, B.J.; Cho, K.S. Stereoobservations of fast magnetosonic waves in the extended solar corona associated with eit/euv waves. Astrophys. J. 2013, 766, 55. [CrossRef]

54. Hariharan, K.; Ramesh, R.K.C.W.T.J. Simultaneous Near-Sun Observations of a Moving Type IV Radio Burst and the Associated White-Light Coronal Mass Ejection. Sol. Phys. 2016, 291. [CrossRef]

55. Patsourakos, S.; Georgoulis, M.K. Near-Sun and 1 AU magnetic field of coronal mass ejections: A parametric study. AEA 2016, 595, A121. [CrossRef]

56. Kooi, J.E.; Fischer, P.D.; Buffo, J.J.; Spangler, S.R. VLA Measurements of Faraday Rotation through Coronal Mass Ejections. Sol. Phys. 2017, 292. [CrossRef]

57. Matsumoto, Y.; Hoshino, M. Onset of turbulence induced by a Kelvin-Helmholtz vortex. Geophys. Res. Lett. 2004, 31. [CrossRef]

58. Zhang, W.; MacFadyen, A.; Wang, P. Three-dimensional relativistic magnetohydrodynamic simulations of the kelvin-helmholtz instability: Magnetic field amplification by a turbulent dynamo. Astrophys. J. 2009, 692, L40-L44. [CrossRef]

59. Murphy, N.A.; Raymond, J.C.; Korreck, K.E. Plasma heating during a coronal mass ejection observed by thesolar and heliospheric observatory. Astrophys. J. 2011, 735, 17. [CrossRef]

60. Fermi, E.; von Neumann, J. Taylor Instability of Incompressible Liquids. Part 1. Taylor Instability of an Incompressible Liquid. Part 2. Taylor Instability at the Boundary of Two Incompressible Liquids; Technical Report; Los Alamos Scientific Lab.: Los Alamos, NM, USA, 1953. [CrossRef]

61. Livescu, D. Compressibility effects on the Rayleigh-Taylor instability growth between immiscible fluids. Phys. Fluids 2004, 16, 118-127. [CrossRef]

(c) 2019 by the authors. Licensee MDPI, Basel, Switzerland. This article is an open access article distributed under the terms and conditions of the Creative Commons Attribution (CC BY) license (http://creativecommons.org/licenses/by/4.0/). 